\section{Excesso de peso de escolares em região do Nordeste Brasileiro: contraste entre as redes de ensino pública e privada}

\section{Excess weight in children from Brazilian Northeast: difference between public and private schools}

Lana do Monte Paula Brasil ${ }^{1}$

Mauro Fisberg ${ }^{2}$

Hélcio de Sousa Maranhão ${ }^{3}$

1,3 Programa de Pós-Graduação em Ciências da Saúde. Departamento de Pediatria. Universidade Federal do Rio Grande do Norte. Rua Gal. Cordeiro de Farias, s.n. Natal, RN, Brasil. CEP: 59.012-570. E-mail: lanabrasil@terra.com.br

2 Departamento de Pediatria. Universidade Federal de São Paulo São Paulo, SP, Brasil

\begin{abstract}
Objectives: to determine the prevalence of excess weight in schoolchildren from the city of Natal.

Methods: transversal study with 1927 children aged 6-11 from public and private schools in different zones in the city of Natal, in the State of Rio Grande do Norte, Brazil, and analyze related variables, such as gender, age-group, type of school and city zones. All scholars with a body mass index equal to or greater than the $85^{\text {th }}$ percentile were considered as having excess weight.

Results: the prevalence of excess weight among the studied scholars was $33.6 \%$. There were no significant differences between genders and age-groups. In the private schools, the prevalence of excess weight was $54.5 \%$, while in the public schools, $15.6 \%(p<0.01$; $O R=6.49$ ). The prevalence of excess weight was greater in schools located in the zones with the best quality of life, east-south zone (41.3\%), if compared with schools in the north-west zone $(28.4 \%)(p<0.01)$.

Conclusions: the prevalence of excess weight in scholars was found high, demonstrating the importance of programs of treatment and prevention. The biggest prevalence in children studying in private schools and in children studying in schools located in the best zone of the city reflects the importance of association between the best socioeconomic levels and these entities in regions of developing countries.
\end{abstract}

Key words Prevalence, Obesity, Child

\section{Resumo}

Objetivos: estimar a prevalência de excesso de peso em escolares na cidade de Natal.

Métodos estudo transversal com 1927 crianças, de 6 a 11 anos de idade, de escolas públicas $e$ privadas nas diferentes zonas da cidade de Natal, Rio Grande do Norte, Brasil. Foram analisadas as variáveis: sexo, faixa etária, tipo de escola e zonas da cidade. Foram considerados com excesso de peso os escolares com índice de massa corporal para sexo e idade igual ou superior ao percentil 85.

Resultados: o excesso de peso foi encontrado em 33,6\% das crianças. Não houve diferenças significantes entre os sexos e faixas etárias. Nas escolas privadas, a prevalência de excesso de peso foi $54,5 \%$; nas públicas, $15,6 \%(p<0,01 ; O R=6,49)$. Maior prevalência de excesso de peso foi encontrada nas escolas das zonas de melhor índice de qualidade de vida da cidade, isto é, zonas leste-sul (41,3\%), quando comparadas às zonas norte-oeste $(28,4 \%)$ $(p<0,01)$.

Conclusões: a prevalência de excesso de peso em escolares se mostrou alta, demonstrando a necessidade de programas de intervenção e prevenção. A maior prevalência nas escolas privadas, reforçada pelo mesmo achado nas crianças de escolas situadas nas zonas de maior poder aquisitivo da cidade, reflete a importância da associação entre os níveis socioeconômicos mais altos e o excesso de peso, sobretudo o sobrepeso, em regiões em desenvolvimento.

Palavras-chave Prevalência, Obesidade, Criança 
Introdução

A obesidade é resultante do desequilíbrio crônico entre a energia ingerida e a utilizada, de origem multifatorial, como as influências ambientais e o componente genético, não sendo ainda possível a identificação da potencialidade de cada um desses fatores e a interação entre eles. ${ }^{1-3}$ Dado que o patrimônio genético humano não mudou em tempos recentes, têm-se considerado a "dieta ocidental", caracterizada pelo alto consumo em gordura, açúcares refinados e pouca fibra, juntamente com a diminuição da atividade física, como fator contribuinte para esse processo. ${ }^{4,5}$

A realidade atual tem demonstrado aumento considerável da prevalência da obesidade nos países em desenvolvimento. ${ }^{6}$ Nesses, o excesso de peso é prevalente nas classes econômicas mais altas, ${ }^{7}$ demonstrando como o fator socioeconômico interfere no seu aparecimento. A transição nutricional pela qual o Brasil passa é constatada pelo aumento progressivo da obesidade em substituição à desnutrição, acontecendo mais rapidamente na faixa etária adulta que na pediátrica. ${ }^{8}$

Segundo Hedley et al., ${ }^{9}$ dados do National Health and Nutrition Examination Survey (NHANES), coletados nos EUA, em 1999-2002, na faixa etária de 6 a 19 anos, determinaram a ocorrência de excesso de peso em $47 \%$ dessa população, mantendo a obesidade como um de seus principais problemas de saúde pública. No Brasil, o modelo da prevalência mundial está se reproduzindo, como identificado na segunda etapa da Pesquisa de Orçamentos Familiares realizado pelo Instituto Brasileiro de Geografia e Estatística (IBGE), ${ }^{10}$ onde se encontrou excesso de peso em $40,6 \%$ da população. Na faixa etária pediátrica, estudos nacionais demonstram prevalências de excesso de peso que variam entre $10,8 \%$ a $33,8 \%$ em diferentes regiões. ${ }^{7,11-22} \mathrm{Na}$ região Nordeste do Brasil, onde a desnutrição infantil outrora ganhava bastante destaque, os estudos são limitados a segmentos específicos e a distintas faixas etárias, além de utilizarem diferentes indicadores nutricionais, dificultando uma boa avaliação. ${ }^{7,12-17,21}$

Nesse contexto, torna-se relevante investigar tal condição, a fim de se obter um melhor conhecimento da freqüência de excesso de peso na faixa etária citada nessa região, o que pode retratar condições semelhantes àquelas encontradas em países em desenvolvimento, por ser a região considerada de menor padrão econômico do país. Portanto, o estudo tem como objetivo estimar a prevalência de excesso de peso em escolares da rede de ensino pública e privada da cidade de Natal.

\section{Métodos}

Realizou-se estudo transversal em amostra de estudantes das redes de ensino pública e privada, no período de setembro de 2002 a abril de 2004, distribuídos nas zonas norte, sul, leste e oeste da cidade de Natal, capital do Estado do Rio Grande do Norte, no Nordeste do Brasil.

Foi utilizado o Indice de Massa Corporal (IMC) $\left(\mathrm{Kg} / \mathrm{m}^{2}\right)$ para classificação em "risco de sobrepeso" e "sobrepeso", de acordo com os pontos de corte sugeridos pelo Center for Disease Control (CDC) and prevention, em 2000. ${ }^{23}$ Para os indivíduos com IMC para idade e sexo $\geq$ percentil 85 , é utilizado o termo "risco de sobrepeso" e, para aqueles com IMC $\geq$ percentil 95 para idade e sexo, o termo "sobrepeso". Considerou-se como "excesso de peso" todas as crianças com risco de sobrepeso ou sobrepeso. O IMC foi calculado e processado pelo software NutStat que utiliza os padrões de referências do CDC 2000. Priorizou-se a análise dos dados baseada na avaliação do grupo com excesso de peso, ou seja, crianças com IMC $\geq$ percentil 85 somadas àquelas $\geq$ percentil 95 para idade e sexo. Posteriormente, analisou-se especificamente o grupo de sobrepeso.

Para o cálculo do tamanho da amostra, levantouse a prevalência de excesso de peso em estudos com crianças no país, porém foram observadas grandes diferenças em relação à faixa etária investigada, aos critérios metodológicos e à região estudada, não sendo possível utilizá-los como a prevalência estimada. Portanto, houve a necessidade de se realizar estudo piloto, observando-se as características da população de escolares da cidade. Dados fornecidos pela Secretaria Municipal de Educação, em 2002, demonstravam em torno de 64.012 estudantes matriculados entre a primeira e quinta série do ensino fundamental, sendo $79 \%$ de escolas públicas e $21 \%$ de escolas privadas. A partir de estudo piloto constituído de 300 crianças, de escolas públicas e privadas, a prevalência de excesso de peso encontrada nessa amostra foi de aproximadamente $25 \%$, sendo essa a estimativa adotada para o cálculo amostral. Considerou-se a margem de erro de $20 \%$, a taxa de não resposta de $20 \%$, o efeito do desenho de 1,5 e um intervalo de confiança de $95 \%$, obtendo-se o total de 2020 crianças.

Realizou-se a amostragem por conglomerados, por sorteio de escolas, no sentido de se facilitar a coleta, tendo em vista que todas as regiões da cidade foram contempladas: zona norte, leste e sul - uma escola pública e duas privadas por zona; zona oeste duas públicas e uma privada, resultando em 1927 
escolares de cinco escolas públicas e sete privadas.

As medidas antropométricas foram realizadas pelos autores e por equipe de estudantes do curso de medicina da Universidade Federal do Rio Grande do Norte, previamente treinados. O peso foi verificado utilizando-se balança digital marca Plenna Lithium, modelo sport, com capacidade máxima de $150 \mathrm{~kg}$ e sensibilidade de $0,1 \mathrm{~kg}$, estando as crianças em uso de roupa de atividade física. A estatura foi aferida com as crianças descalças, eretas, utilizando-se estadiômetro portátil marca Seca, modelo 206, com fita de aço graduada até $220 \mathrm{~cm}$ e divisões em milímetros, respeitando normas recomendadas. ${ }^{24}$

As variáveis analisadas foram: sexo, faixa etária (faixa I: crianças $\geq 6$ anos e $<9$ anos, faixa II: crianças $\geq 9$ anos e $<11$ anos), tipo de escola e zona da cidade, onde foram agrupadas as zonas leste e sul, comparando-as com o agrupamento das zonas norte e oeste. Essas associações de zonas (leste-sul e norte-oeste) respeitaram as semelhanças encontradas no estudo "Mapeando a Qualidade de Vida em Natal", realizado em 2003, onde as zonas leste e sul demonstraram índice de qualidade de vida superior às zonas norte e oeste. ${ }^{25}$

Para a comparação das prevalências de excesso de peso e sobrepeso entre as variáveis, foi utilizado o teste do qui-quadrado com correção de Yates, nível de significância de $p<0,05$. Também foi determinado o OR e intervalo de confiança de $95 \%$ (IC95\%).

A análise estatística dos dados foi realizada através do software Epi-Info, versão 3.3 de 2004 do Center for Disease Control e com a supervisão técnica do Núcleo de Desenvolvimento do Tirocínio Científico da Faculdade de Odontologia da Universidade Federal do Rio Grande do Norte.

O projeto foi aprovado pelo Comitê de Ética em Pesquisa da Universidade Federal do Rio Grande do Norte.

\section{Resultados}

Das 1927 crianças, $985(51,1 \%)$ eram do sexo masculino e $942(48,9 \%)$ do feminino, $1084(56,2 \%)$ da faixa etária I e $843(43,8 \%)$ da faixa etária II, 895 $(46,4 \%)$ de escolas privadas e $1032(53,6 \%)$ de públicas, $787(40,8 \%)$ da zona leste-sul e 1140 $(59,2 \%)$ da zona norte-oeste.

$\mathrm{Na}$ amostra, o excesso de peso foi encontrado em $33,6 \%$ (649 crianças), enquanto o sobrepeso foi encontrado em $22,6 \%$ (436 crianças), conforme mostra Tabela 1.

$\mathrm{Na}$ Tabela 2 foram observadas as prevalências de excesso de peso e sobrepeso nos sexos masculino $(35,4 \%$ e $23,0 \%$, respectivamente) e feminino ( $31,8 \%$ e $22,2 \%$, respectivamente), não sendo encontradas diferenças significantes. Também não foram observadas diferenças significantes entre excesso de peso e sobrepeso em qualquer das faixas etárias I $(31,8 \%$ e $21,1 \%$, respectivamente) e II $(36,1 \%$ e

\section{Tabela 1}

Prevalência de baixo peso, eutrófico, risco de sobrepeso e sobrepeso em 1927 escolares das redes pública e privada de ensino. Natal, Rio Grande do Norte.

\begin{tabular}{lcc}
\hline & \multicolumn{2}{c}{ Prevalência } \\
\cline { 2 - 3 } Estado nutricional & $\mathrm{n}$ & $\%$ \\
\hline Baixo peso (IMC <percentil 5) & 113 & 5,9 \\
Eutrófico (percentil 5 $\leq$ IMC <percentil 85) & 1165 & 60,5 \\
Excesso de peso (IMC $\geq$ percentil 85) & 649 & 33,6 \\
Risco para sobrepeso (percentil 85 $\leq$ IMC < percentil 95) & 213 & 11,0 \\
Sobrepeso (IMC $\geq$ percentil 95) & 436 & 22,6 \\
Total & 1927 & 100,0 \\
\hline
\end{tabular}

IMC=índice de massa corporal. 
$24,5 \%$, respectivamente), como se pode constatar na Tabela 3.

Ao analisar as escolas públicas e privadas (Tabela 4), observou-se que do total de 895 alunos das escolas privadas, $488(54,5 \%)$ apresentavam excesso de peso e 383 (42,8\%) sobrepeso. Dos 1032 alunos das escolas públicas, foram encontrados 161 $(15,6 \%)$ e $53(5,1 \%)$ com excesso de peso e sobrepeso, respectivamente. Esses percentuais apre- sentaram grande diferença estatística $(p<0,01)$, tanto para o excesso de peso como para sobrepeso, com odds ratio (OR) igual a 6,49 e 13,8 , respectivamente.

Na Tabela 5, observa-se que as escolas da zona leste e sul demonstraram significantemente $(p<0,01)$ maior prevalência de excesso de peso $(41,3 \%)$ e sobrepeso $(29,7 \%)$ do que a zona norte e oeste $(28,4 \%$ e $17,9 \%$, respectivamente).

Tabela 2

\begin{tabular}{lccccccccc}
\hline Prevalência de excesso de peso e sobrepeso em escolares de acordo com o sexo. Natal, Rio Grande do Norte. \\
\hline
\end{tabular}

* Teste do qui-quadrado: $p=0,10 ;{ }^{* *}$ Teste do qui-quadrado: $p=0,69$.

Tabela 3

Prevalência de excesso de peso e sobrepeso em escolares de acordo com a faixa etária. Natal, Rio Grande do Norte.

\begin{tabular}{|c|c|c|c|c|c|c|c|c|c|c|}
\hline \multirow{3}{*}{ Faixa etária } & \multirow{2}{*}{\multicolumn{2}{|c|}{ Total }} & \multicolumn{4}{|c|}{$\begin{array}{l}\text { Excesso de peso* } \\
I M C \geq \text { percentil } 85\end{array}$} & \multicolumn{4}{|c|}{$\begin{array}{c}\text { Sobrepeso }{ }^{\star *} \\
I M C \geq \text { percentil } 95\end{array}$} \\
\hline & & & \multicolumn{2}{|c|}{$\operatorname{sim}$} & \multicolumn{2}{|c|}{ não } & \multicolumn{2}{|c|}{$\operatorname{sim}$} & \multicolumn{2}{|c|}{ não } \\
\hline & $\mathrm{n}$ & $\%$ & $\mathrm{n}$ & $\%$ & $\mathrm{n}$ & $\%$ & $\mathrm{n}$ & $\%$ & $\mathrm{n}$ & $\%$ \\
\hline Faixa etária I ( $\geq 6$ e $<9$ anos) & 1084 & 56,2 & 345 & 31,8 & 739 & 68,2 & 229 & 21,1 & 855 & 78,9 \\
\hline Faixa etária II ( $\geq 9$ e $<11$ anos) & 843 & 43,8 & 304 & 36,1 & 539 & 63,9 & 207 & 24,5 & 636 & 75,5 \\
\hline Total & 1927 & 100,0 & 649 & 33,6 & 1278 & 66,4 & 436 & 22,6 & 1491 & 77,4 \\
\hline
\end{tabular}

* Teste do qui-Quadrado: $p=0,05 ;{ }^{* *}$ Teste do qui-quadrado: $p=0,08$. 
Prevalência de excesso de peso e sobrepeso em escolares de acordo com o tipo de escola. Natal, Rio Grande do Norte.

\begin{tabular}{|c|c|c|c|c|c|c|c|c|c|c|}
\hline \multirow{3}{*}{ Escolas } & \multirow{2}{*}{\multicolumn{2}{|c|}{ Total }} & \multicolumn{4}{|c|}{$\begin{array}{l}\text { Excesso de peso* } \\
\text { IMC } \geq \text { percentil } 85\end{array}$} & \multicolumn{4}{|c|}{$\begin{array}{c}\text { Sobrepeso** } \\
\text { IMC } \geq \text { percentil } 95\end{array}$} \\
\hline & & & \multicolumn{2}{|c|}{$\operatorname{sim}$} & \multicolumn{2}{|c|}{ não } & \multicolumn{2}{|c|}{$\operatorname{sim}$} & \multicolumn{2}{|c|}{ não } \\
\hline & $\mathrm{n}$ & $\%$ & $\mathrm{n}$ & $\%$ & $\mathrm{n}$ & $\%$ & $\mathrm{n}$ & $\%$ & $\mathrm{n}$ & $\%$ \\
\hline Pública & 1032 & 53,6 & 161 & 15,6 & 871 & 84,4 & 53 & 5,1 & 979 & 94,9 \\
\hline Privada & 895 & 46,4 & 488 & 54,5 & 407 & 45,5 & 383 & 42,8 & 512 & 57,2 \\
\hline Total & 1927 & 100,0 & 649 & 33,6 & 1278 & 66,4 & 436 & 22,6 & 1491 & 77,4 \\
\hline
\end{tabular}

* Teste do qui-quadrado: $p<0,01$; OR=6,49; Intervalo de confiança 95\%: 5,21 - 8,08; ${ }^{* *}$ Teste do qui-quadrado: $p<0,01$; $\mathrm{OR}=13,8$; Intervalo de confiança $95 \%$ : 10,0-19,0.

Tabela 5

Prevalência de excesso de peso e sobrepeso em escolares da acordo com a localização das escolas nas zonas da cidade de Natal, Rio Grande do Norte.

\begin{tabular}{|c|c|c|c|c|c|c|c|c|c|c|}
\hline \multirow{3}{*}{ Zonas } & \multirow{2}{*}{\multicolumn{2}{|c|}{ Total }} & \multicolumn{4}{|c|}{$\begin{array}{l}\text { Excesso de peso } \\
\text { IMC } \geq \text { percentil } 85\end{array}$} & \multicolumn{3}{|c|}{$\begin{array}{c}\text { Sobrepeso } \\
\text { IMC } \geq \text { percentil } 95\end{array}$} & \\
\hline & & & \multicolumn{2}{|c|}{$\operatorname{sim}$} & \multicolumn{2}{|c|}{ não } & \multicolumn{2}{|c|}{$\operatorname{sim}$} & \multicolumn{2}{|c|}{ não } \\
\hline & $n$ & $\%$ & $\mathrm{n}$ & $\%$ & $n$ & $\%$ & $n$ & $\%$ & $n$ & $\%$ \\
\hline Leste-sul & 787 & 40,8 & 325 & 41,3 & 462 & 58,7 & 234 & 29,7 & 553 & 70,3 \\
\hline Norte-oeste & 1140 & 59,2 & 324 & 28,4 & 816 & 71,6 & 202 & 17,9 & 938 & 82,1 \\
\hline Total & 1927 & 100,0 & 649 & 33,6 & 1278 & 66,4 & 436 & 22,6 & 1491 & 77,4 \\
\hline
\end{tabular}

* Teste do qui-quadrado: $p<0,01 ; \mathrm{OR}=1,77$; Intervalo de confiança 95\%: 1,46-2,16; ** Teste do qui-quadrado: $p<0,01 ; \mathrm{OR}=1,96$; Intervalo de confiança $95 \%: 1,57-2,45$.

\section{Discussão}

O aumento progressivo da obesidade infantil é uma preocupação de saúde pública. Há evidências de sua associação com entidades crônico-degenerativas na fase adulta. ${ }^{2}$ Por ser uma doença de difícil controle, a preocupação na sua prevenção em fase precoce da vida é uma medida que pode promover grandes benefícios.

A falta de padronização na definição de termos, nas variáveis antropométricas e respectivos pontos de corte relacionados a sobrepeso e obesidade na infância e adolescência traz dificuldades nas comparações de estudos de prevalências. Nesse sentido, fez-se a opção de se utilizar a padronização recomendada pelo CDC, ${ }^{23}$ em 2000. Deve-se levar em conta que essa classificação traz polêmica por determinar como "sobrepeso" àqueles com IMC acima do percentil 95. Características sociais, políticas e de proteção à infância tentam justificar essa condição. No entanto, em países em desenvolvimento, admite-se chamar de "obesos" todos os indivíduos aí incluídos por haver clara associação entre o excesso de massa corporal e o excesso de massa gordurosa. Como não foi avaliada qualquer medida de massa, optou-se pela classificação do CDC, e não aquela de Must et al., ${ }^{26}$ em que a denominação sobrepeso é dada para pessoas com IMC acima do percentil 85 e obesidade para IMC acima do percentil 95 .

Os estudos de prevalência em crianças com excesso de peso, tanto nacionais como interna- 
cionais, demonstram percentuais extremamente variáveis, na dependência da clientela analisada, do nível socioeconômico e da faixa etária, condições essas intervenientes nestes valores e de difíceis comparações. Em Natal, Lyra et al., ${ }^{27}$ estudando 562 adolescentes de ambos os sexos de escolas públicas, encontraram $17,1 \%$ com IMC $\geq$ percentil 90 para os respectivos sexos e idades. No atual estudo, a prevalência de $33,6 \%$ pode ser considerada alta, quando comparada aos percentuais encontrados por Ramos e Barros Filho, ${ }^{11}$ em Bragança Paulista, São Paulo (10,8\%), Oliveira et al., ${ }^{15}$ em Feira de Santana-Bahia (13,7\%), Motta e Silva, ${ }^{14}$ em Recife, Pernambuco (14,7\%) e Abrantes et al., ${ }^{17}$ em cidades das regiões Nordeste e Sudeste (17,3\%), porém todos esses autores analisaram casuísticas em faixas etárias pediátricas diferentes, com distintos pontos de corte de IMC. Por outro lado, assemelha-se ao estudo de Costa et al., ${ }^{20}$ que encontraram prevalência de 33,7\% em escolares de 7 a 10 anos, em Santos-São Paulo. Chama a atenção o fato de que o estudo ora apresentado reflete a prevalência de excesso de peso em uma capital de Estado situada no Nordeste, região onde as condições socioeconômicas são as mais desfavoráveis do país. ${ }^{10}$

Do total da amostra, foram encontradas $22,6 \%$ de crianças com IMC $\geqq$ percentil 95, demonstrando uma maior contribuição dos sobrepesos no percentual do excesso de peso, do que as crianças com risco de sobrepeso (percentil $85 \leqq$ IMC <percentil 95) (11\%), apresentando divergência dos resultados habitualmente encontrados na literatura, ${ }^{7,11-15,17,18}$ mas que se assemelham aos dados de Fonseca et al. ${ }^{19}$ e aos de Costa et al. ${ }^{20}$ Isso traz maiores preocupações em virtude de as crianças já comportarem condições mais avançadas de excesso de peso.

Quanto ao sexo, não foram encontradas diferenças significantes entre as prevalências de excesso de peso e de sobrepeso, aproximando-se dos resultados de alguns estudos. ${ }^{7,15,18}$ Porém, Balaban e Silva, ${ }^{12}$ em Recife, Pernambuco, e Soar et al., ${ }^{22}$ em Florianópolis, Santa Catarina, encontraram maiores percentuais de excesso de peso no sexo masculino. Outros autores demonstraram maior prevalência no sexo feminino. ${ }^{13,16,17}$

A estratificação da casuística em duas faixas etárias permitiu observar que não houve diferença significante entre a primeira e a segunda faixa, mas notam-se valores com forte tendência à significância tanto para excesso de peso $(p=0,05)$ quanto para sobrepeso $(p=0,08)$, com maiores percentuais na Faixa II ( $\geqq 9$ e $<11$ anos). Esses achados podem ser justificados pelo maior incremento do IMC já no início da puberdade, secundário ao aumento da massa corporal. As crianças não foram avaliadas quanto ao estadiamento puberal, mas considera-se aceitável que nessa faixa etária tais eventos possam estar presentes, influindo assim na sua composição corporal.

A análise das crianças distribuídas em escolas públicas e privadas permite avaliar a importância do nível socioeconômico na determinação do excesso de peso. Neste estudo, a maior prevalência de excesso de peso e sobrepeso foi observada nas escolas privadas, sendo encontrados altos percentuais $(54,5 \%$ e $42,8 \%$, respectivamente), enquanto nas escolas públicas tais percentuais foram $15,6 \%$ e $5,7 \%$, respectivamente. Portanto, as maiores prevalências nas escolas privadas contribuíram de forma mais efetiva para os achados de toda casuística. Em regiões de melhor padrão socioeconômico, Costa et al. ${ }^{20}$ na região Sudeste do Brasil, e Giugliano e Carneiro ${ }^{18}$ na região Centro-Oeste do Brasil, encontraram resultados semelhantes. É importante ressaltar que, no presente estudo, as crianças de escolas privadas demonstraram risco de sobrepeso 13,8 vezes mais do que as crianças de escolas públicas. Além disso, mais da metade das crianças de escolas privadas encontrava-se com excesso de peso. Isso faz realçar a maior importância do foco da atenção no controle e prevenção nas escolas privadas. Ratificando esses achados, a análise entre as diferentes zonas da cidade demonstrou haver maior prevalência de excesso de peso e sobrepeso em alunos matriculados nas escolas das zonas consideradas de melhor qualidade de vida. Tais resultados retratam o modelo encontrado em países em desenvolvimento, onde o excesso de peso ainda é predominante em melhores condições socioeconômicas, refletindo a chamada transição nutricional. ${ }^{8}$ No Brasil, a relação positiva entre os mais altos níveis socioeconômicos e obesidade começa a apresentar inversão, assemelhando-se aos países desenvolvidos, como mostram os levantamentos de Monteiro et al. ${ }^{28}$ e Batista Filho e Rissin ${ }^{8}$ em adultos. No entanto, a casuística ora apresentada certamente ainda foge a essa nova situação. Para tal ocorrência justifica-se, na literatura, que as classes socioeconômicas altas teriam maior disponibilidade de alimentos, fazendo uso inadequado desses, e sofreriam mais as influências do sedentarismo imposto pelo padrão de vida moderno. ${ }^{2}$ Percebe-se, portanto, a realidade dessas informações na população estudada.

A prevenção do excesso de peso na infância leva o pediatra para o topo da cadeia de profissionais que trabalham com esses distúrbios. O espaço escolar 
mostra-se um ambiente propício para as intervenções preventivas, pois aí se encontra o grande percentual de crianças, viabilizando as estratégias de melhoria na educação nutricional. A atuação preventiva nessa faixa promove a diminuição de doenças relacionadas na população adulta.

A prevalência de excesso de peso e sobrepeso em escolares se mostrou alta na região investigada, demonstrando a necessidade de seu controle e prevenção. Nesse meio, obedecendo ao modelo de países em desenvolvimento, observa-se a grande influência da condição socioeconômica, demonstrada pelos altos percentuais de excesso de peso em crianças de escolas privadas.

\section{Referências}

1. Fisberg M. Primeiras palavras: uma introdução ao problema do peso excessivo. In: Fisberg M, editor. Atualização em obesidade na infância e adolescência. São Paulo: Atheneu; 2004. p. 1-9.

2. Speiser PW, Rudolf MCJ, Anhalt H, Camacho-Hubner C, Chiarelli F, Eliakim A, Freemark M, Gruters A, Hershkovitz E, Iughetti L, Krude H, Latzer Y, Lustig RH, Pescovitz OH, Pinhas-Hamiel O, Rogol AD, Shalitin S, Sultan C, Stein D, Vardi P, Werther GA, Zadik Z, Zucherman-Levin N, Hochberg Z. Concensus statement: chilhood obesity. J Clin Endocrinol Metab. 2005; 90: 1871-87.

3. Ebbeling CB, Pawlak DB, Ludwig DS. Childhood obesity: public-health crisis, common sense cure. Lancet. 2002; 360: 473-82.

4. Francis LA, Lee Y, Birch LL. Parental weight status and girls'television viewing, snacking, and body mass index. Obes Res. 2003; 11: 143-51

5. Monteiro CA, Mondini L, Souza ALM, Popkin B. Da desnutrição para a obesidade: a transição nutricional no Brasil. In: Monteiro CA, organizador. Velhos e novos males da saúde no Brasil: a evolução do país e de suas doenças. 2. ed. aum. São Paulo: Hucitec; 2000. p. 247-55.

6. Caballero B. Introduction. Symposium: Obesity in developing countries: biological and ecological factors. J Nutr. 2001; 131: S866-70.

7. Balaban G, Silva GAP, Motta MEFA. Prevalência de sobrepeso e obesidade em escolares de diferentes classes socioeconômicas em Recife, Pernambuco. Pediatria. (São Paulo) 2001; 23: 285-9.

8. Batista Filho M, Rissin A. A transição nutricional no Brasil: tendências regionais e temporais. Cad Saúde Pública. 2003; 19 (Supl 1): 181-91.

9. Hedley AA, Ogden CL, Johnson CL, Carroll MD, Curtin LR, Flegal KM. Prevalence of overweight and obesity among US children, adolescents and adults, 1999-2002. JAMA. 2004; 291: 2847-50.

10. IBGE (Instituto Brasileiro de Geografia e Estatística). Pesquisa de Orçamentos Familiares: 2002-2003. Disponível em URL: www.ibge.gov.br [2006 set 15].

\section{Agradecimentos}

Aos professores Ângelo Giuseppe Roncalli da Costa Oliveira e Maria Angela Ferreira, pela orientação estatística. Aos acadêmicos do curso médico Elida Cristina de Medeiros, Judith Marques Duarte Cunha, Juliana Carvalho Rodrigues, Juliana Costa Reis, Liana Berucia Freire de Oliveira, Priscilla Vargas Waslsh G dos Santos, Railson Andrielle Silva Brandão, Raissa Anielle Silva Brandão, Richardson Ozhéas Bezerra Campos e Sesionne Maciel da Silveira, pela ajuda imprescindível na coleta de dados.
11. Ramos AMPP, Barros Filho AA. Prevalência de obesidade em adolescentes de Bragança Paulista e sua relação com obesidade dos pais. Arq Bras Endocrinol Metab. 2003; 47: 677-83.

12. Balaban G, Silva GAP. Prevalência de sobrepeso e obesidade em crianças e adolescentes de uma escola da rede privada de Recife. J Pediatr. (Rio J). 2001; 77: 96-100.

13. Silva GAP, Balaban G, Freitas MMV, Baracho JDS, Nascimento EMM. Prevalência de sobrepeso e obesidade em crianças pré-escolares matriculadas em duas escolas particulares de Recife, Pernambuco. Rev Bras Saúde Matern Infant. 2003; 3: 323-7.

14. Motta MEFA, Silva GAP. Desnutrição e obesidade em crianças: delineamento do perfil de uma comunidade de baixa renda. J Pediatr. (Rio J) 2001; 77: 288-93.

15. Oliveira AMA, Cerqueira EMM, Oliveira AC. Prevalência de sobrepeso e obesidade infantil na cidade de Feira de Santana, Bahia: detecção na família x diagnóstico clínico. J Pediatr. (Rio J) 2003; 79: 325-8.

16. Leão LSCS, Araújo LMB, Moraes LTLP, Assis AM. Prevalência de obesidade em escolares de Salvador, Bahia. Arq Bras Endocrinol Metab. 2003; 47: 151-7.

17. Abrantes MM, Lamounier JA, Colosimo EA. Prevalência de sobrepeso e obesidade nas regiões Nordeste e Sudeste do Brasil. Rev Assoc Med Bras. 2003; 49: 162-6.

18. Giugliano R, Carneiro EC. Fatores associados à obesidade em escolares. J Pediatr. (Rio J) 2004; 80: 17-22.

19. Fonseca VM, Sichieri R, Veiga GV. Fatores associados à obesidade em adolescentes. Rev Saúde Publica. 1998; 32: 541-9.

20. Costa RF, Cintra IP, Fisberg M. Prevalência de sobrepeso e obesidade em escolares da cidade de Santos, SP. Arq Bras Endocrinol Metab. 2006; 50: 60-7.

21. Silva GAP, Balaban G, Motta MEFA. Prevalência de sobrepeso e obesidade em crianças e adolescentes de diferentes condições socioeconômicas. Rev Bras Saúde Matern Infant. 2005; 5: 53-9. 
22. Soar C, Vasconcelos FAG, Assis MAA, Grosseman S, Luna MEP. Prevalência de sobrepeso e obesidade em escolares de uma escola pública de Florianópolis, Santa Catarina. Rev Bras Saúde Matern Infant. 2004; 4: 391-7.

23. Kuczmarski RJ, Ogden CL, Guo SS, Grummer-Strawn LM, Flegal KM, Mei Z, Wei R, Curtin LR, Roche AF, Johnson AL. 2000 CDC Growth Charts for the United States: methods and development. Vital Health Stat. 2002; 246: 1190.

24. WHO (World Health Organization). Expert Committee on Physical Status. The use and interpretation of anthoropometry physical status. Geneva; 1995. (WHO Technical Report Series, v. 854).

25. Barroso AV. Mapeando qualidade de vida em Natal-2003. Disponível em URL: http://www.natal.rn.gov.br/sempla/ index.php . [2006 set 15].
26. Must A, Dallal GE, Dietz WH. Reference data for obesity: 85th and 95th percentiles of body mass index (wt/ht2) and triceps skinfold thickness. Am J Clin Nutr. 1991; 53: 83946.

27. Lyra CO, Mendonça GAS, Sousa IC. Estado nutricional em escolares de Natal, Rio Grande do Norte. Recife: IMIP; 2001. (Publicações Científicas do Instituto Materno Infantil de Pernambuco, n.3).

28. Monteiro CA, Benicio MH, Conde WL, Pookin BM. Shifting obesity trends in Brazil. Eur J Clin Nutr. 2000; 54: 342-6.

Recebido em 3 de novembro de 2006

Versão final apresentada em 20 de junho de 2007

Aprovado em 30 de julho de 2007 